\title{
A dimensão psicossocial na promoção de práticas alimentares saudáveis*
}

Bruna Robba Lara ${ }^{1}$

Vera Silvia Facciolla Paiva²

LARA, B.R.; PAIVA, V.S.F. The psychosocial dimension in promoting healthy dietary practices. Interface - Comunic., Saude, Educ., v.16, n.43, p.1039-54, out./dez. 2012.

This paper critically examines how the psychosocial dimension is dealt with in promoting healthy dietary practices. A search was carried out in Lilacs and in Medline's multipurpose mode, from 2000 to 2011, using the terms intervention, health promotion and psychosocial, and all terms relating to nutrition. It was observed that during this last decade, sociocognitive approaches and models of rational belief still predominated in this field. Intervention studies focusing on the individual prevailed, with less attention paid to the broader social context that produces dietary practices. It can be concluded that for healthy dietary practices to be promoted within the context of comprehensive healthcare, the debate about what can be called psychosocial needs to be broadened to incorporate the recent contributions from healthcare approaches based on human rights, with awareness of the multidimensionality of the health-disease-care process.

Keywords: Health promotion. Psychosocial. Care. Dietary and nutritional education. Human rights.
Este artigo analisa criticamente como é abordada a dimensão psicossocial na promoção de práticas alimentares saudáveis. Realizou-se busca no Lilacs e no modo multipurpose do Medline, de 2000 a 2011, utilizando os termos intervenção, promoção da saúde, psicossocial e todos aqueles correlatos à nutrição. Observou-se que nesta última década as abordagens sociocognitivas e modelos de crença racional ainda predominam nesse campo, prevalecendo trabalhos de intervenção focados no indivíduo e pouco críticos ao contexto social mais amplo que produz práticas alimentares. Conclui-se que para a promoção de práticas alimentares saudáveis no contexto de assistência integral, o debate sobre o que chamamos de psicossocial deve ser ampliado para incorporar as contribuições recentes das abordagens em saúde com base nos direitos humanos, atentas à multidimensionalidade do processo saúde-doença-cuidado.

Palavras-chave: Promoção da saúde. Psicossocial. Cuidado. Educação alimentar e nutricional. Direitos Humanos.

\footnotetext{
- Elaborado com base em Lara (2010)

${ }^{1}$ Núcleo de Estudos para a Prevenção da Aids (NEPAIDS). Av. Prof. Mello Moraes 1721 Bloco A-Sala 117 Cidade Universitária. São Paulo, SP, Brasil 04.560-012. brunarl@usp.br

${ }^{2}$ Departamento de Psicologia Social e do Trabalho, Instituto de Psicologia, Universidade de São Paulo.
} 


\section{Introdução}

A promoção de práticas alimentares saudáveis se configura como relevante questão de saúde global, preocupação de instituições governamentais e profissionais de saúde assim como de indivíduos que sofrem com os efeitos de uma alimentação inadequada. No Brasil o Ministério da Saúde (Brasil, 2010) destaca o crescimento do número de brasileiros com excesso de peso e permanência da desnutrição e doenças por carências de micronutrientes, o que demanda renovar as ações no Sistema Único de Saúde (SUS) nesse campo, em especial na atenção básica.

Ao nos debruçarmos sobre os desafios no campo das práticas alimentares no Brasil, é quase intuitivo compreender que a trajetória pessoal na família, a cultura, como as crianças são socializadas para comer ou deixar de comer e o acesso à mídia são dimensões que participam do processo. São domínios e processos típicos do campo psicossocial, que certamente é um tema caro à promoção de práticas alimentares saudáveis. Em trabalho anterior (Lara, 2010), observou-se que havia mais de uma forma de compreender a dimensão psicossocial na promoção de práticas alimentares saudáveis, diferenças alinhadas a propostas distintas de promoção da saúde.

O termo "psicossocial" é amplamente utilizado na literatura em saúde, embora não seja autoexplicativo. Normalmente, refere-se a ações de atenção ao indivíduo, com uma compreensão de que esse indivíduo participa de, ou está inserido em, um contexto social. Raramente, entretanto, se especifica de que maneira esse termo está sendo utilizado ou a partir de que referencial teórico. Expressões como "suporte psicossocial", "atenção psicossocial", "aspectos psicossociais" são utilizadas sem mais explicações em relação ao posicionamento do autor sobre o que é "psicossocial". Embora seu uso ressalte que o indivíduo vive em sociedade, a interpretação dos problemas e as possibilidades de intervenção dependerão da visão que se tem dessa relação entre indivíduos e sociedade.

Ao mesmo tempo, a dimensão psicossocial em estratégias definidas como de promoção da saúde será compreendida e abordada de maneira diferente se estiver referida às concepções filiadas ao modelo de História Natural da Doença (HND) ou ao que se nomeou como nova ou moderna promoção da saúde (MPS). Em comum, essas duas propostas de promoção da saúde implicam em uma compreensão da multidimensionalidade do processo saúde-doença-cuidado porque outras dimensões, que não a biológica, estariam envolvidas nos processos de adoecimento. Além disso, ambas requerem um abordagem interdisciplinar das questões de saúde e ações para além do setor da saúde.

Por outro lado, enquanto a promoção da saúde no modelo de HND tem uma visão mais naturalizada do processo saúde-doença ${ }^{3}$, a MPS está mais fortemente atenta à dimensão política e social desse processo. Mais ainda, no modelo de HND a promoção da saúde é um dos momentos da prevenção de doenças, consistindo na melhoria geral das condições de vida de indivíduos, famílias e comunidades, beneficiando a saúde e qualidade de vida e tornando-os mais resistentes a processos patogênicos (Ayres, 2009, grifo nosso), ou seja, a saúde é concebida como ausência de doenças, e as estratégias de promoção da saúde adotadas por esse modelo propõem, em geral, "medidas gerais, educativas, que objetivam melhorar a resistência, o bem-estar geral dos indivíduos [...] para que resistam à agressão dos agentes" (Westphal, 2006, p.641), não alterando de fato as condições de vida de indivíduos e comunidades. A MPS, em comparação, deixa de ser um momento na organização de ações de prevenção e passa a ser utilizada como norte de um movimento de renovação das práticas de saúde e da própria

\footnotetext{
${ }^{3}$ Nos anos 1970 a promoção da saúde do modelo de HND passou a ser duramente criticada: a multicausalidade e os determinantes sociais políticos do processo saúde-doença-cuidado teriam sido mal incorporados ao modelo - a própria noção de HND implicaria em um curso "natural" da doença, o que impediria uma compreensão mais politizada dos determinantes do processo saúde-doença-cuidado (Ayres, 2009).
} 
${ }^{4}$ WORLD HEALTH ORGANIZATION. WHO. Health promotion glossary. WHO: Geneve, 1998. Disponível em: <http://www.ldb.org/vl/ top/glossary.pdf $>$. Acesso em: 9 jun. 2012. definição de saúde, referindo-se a todas as fases e níveis do modelo de HND, reconstruindo suas bases filosóficas e métodos (Ayres, 2009).

Em 1998 a OMS definiu saúde na perspectiva da promoção da saúde como recurso à vida individual, social e econômica ${ }^{4}$ (Traverso-Yépes, 2007). Ou seja, não se trata apenas de prevenir e evitar doenças, porque a saúde deixa de ser um fim em si mesmo, para compor um recurso da vida cotidiana. A MPS traz uma compreensão dinâmica dos determinantes do processo saúde-doença-cuidado (Sícoli, Nascimento, 2003): determinantes sociais, econômicos, ambientais e biológicos compõem um processo sinérgico, complexo, e sua ação só pode ser compreendida em suas interconexões. Além disso, evocando processos de transformação social para além de atividades educativas voltadas para a prevenção de doenças, o princípio da equidade é incorporado, bem como a participação social, empoderamento individual e coletivo e a sustentabilidade das ações. Quando a MPS procura envolver as pessoas como parceiros na busca por melhores condições de vida, altera-se o sentido da promoção da saúde no modelo de HND, em que as pessoas são "público-alvo" de ações focadas na prevenção de doenças.

Assim, embora tanto a promoção da saúde no modelo de HND como no da MPS permitam falar em uma dimensão psicossocial do processo saúde-doençacuidado, essa dimensão será abordada de maneira diferente em cada uma dessas propostas de promoção da saúde. Cientes dessas diferenças, as autores do presente trabalho fazem uma revisão de intervenções que levam em conta a dimensão psicossocial na promoção de práticas alimentares saudáveis indexadas no Medline e Lilacs a fim de identificar as diferentes concepções de dimensão psicossocial presentes nessas intervenções. Finalmente, são feitos alguns apontamentos em relação aos modelos de promoção da saúde com os quais cada uma dessas concepções dialogam.

\section{Duas vertentes para pensar a dimensão psicossocial}

Spink (1992) discutia que os estudos que mais frequentemente abordam a dimensão psicossocial no campo da saúde poderiam ser categorizados em duas grandes vertentes, uma que enfatiza o estilo de vida na cadeia multicausal responsável pelo surgimento da doença e outra, construcionista, que compreende o processo saúde-doença-cuidado como um fenômeno psicossocial, historicamente construído.

Ainda hoje a leitura de livros clássicos do campo da psicologia da saúde, por exemplo, permitiria identificar que há predomínio da primeira dessas vertentes, que tem por foco o indivíduo e busca promover alterações cognitivocomportamentais para favorecer comportamentos que poderiam deter ou atenuar processos de adoecimento. Destacam-se nessa vertente os modelos "Health Belief Model" - HBM (ver Strecher, Rosenstock, 2002), "Theory of Planned Behavior e Theory of Reasoned Action" - TPB e TRA (ver Montaño, Kasprzyk, Taplin, 2002) e o "Transtheoretical Model" - TM (ver Prochaska, Redding, Evers, 2002). Esses modelos baseiam-se no pressuposto de que as percepções, crenças e conhecimentos dos indivíduos contribuem - em graus variados - para comportamentos ou hábitos que expõem ao risco em saúde (Tunala-Mendonça, 2005; Crossley, 2000). Valorizam crenças e pensamentos relacionados à saúde e à percepção sobre o risco, bem como a motivação para a mudança, autoconfiança, a aquisição de estratégias, conhecimentos e habilidades que favoreçam a adoção de comportamentos protetores.

A segunda vertente, a vertente de inspiração construcionista (Paiva, Ayres, Gruskin, 2010; Paiva et al., 2010; Benzaken et al., 2007; Paiva, 2005; Paiva, Ayres, 
França-Jr, 2004; Spink, 1992) e que se aproximou do referencial dos Direitos Humanos em saúde (Ayres, Paiva, França Júnior, 2011; Santos, 2011; Paiva, 2010; Ayres et al., 2006; Mann, Tarantola, 1996), tem desenvolvido programas de promoção da saúde integral junto a diferentes comunidades e segmentos da população, e aparece fortemente em artigos e capítulos no campo da chamada Resposta Brasileira à Aids ou do Programa de Atenção à Saúde da Mulher (PAISM) (Berkman et al., 2005). Não discriminação e participação de usuários são princípios centrais dessas abordagens, assim como o princípio do acesso universal, da aceitabilidade e qualidade das ações em saúde, assegurando que as populações socialmente mais vulneráveis consigam ser atendidas em suas necessidades de saúde (Gruskin, Tarantola, 2008).

A vertente focada na mudança de estilos de vida dialoga com a promoção da saúde característica do modelo de HND, uma vez que se baseia principalmente em medidas educativas e tem como finalidade evitar o adoecimento. A segunda vertente, construcionista, alinha-se à MPS. Essa vertente busca transformar as condições objetivas de vida, concebendo saúde como recurso à vida cotidiana, e não como ausência de doença. Mais ainda, reconhece a necessidade de transformações sociais e políticas, considerando que o processo saúde-doença não é natural, mas atravessado por essas questões, ressaltando a necessidade de ações programáticas e sustentáveis para enfrentar a iniquidade e garantir melhores condições de vida para a população. Assim como na proposta da MPS, que compreende os determinantes do processo saúde-doença de maneira dinâmica, a vertente construcionista também compreende a dimensão psicossocial desse processo como interação entre dimensões mutuamente implicadas: a dimensão individual, social e programática. Como proposta de ação, essa vertente atua capacitando indivíduos e comunidades para que reconheçam necessidades e aspirações e atuem no sentido de buscar as modificações necessárias, de maneira semelhante ao que propõe a MPS.

\section{A literatura sobre promoção de práticas alimentares saudáveis}

A maior parte dos artigos disponíveis sobre a promoção de práticas alimentares saudáveis se alinhava à vertente focada na mudança de estilos de vida, propondo intervenções de base sociocognitiva e comportamental, que assumem que as percepções, crenças e conhecimento contribuem em graus variados para a performance (ou não) de determinado comportamento (Crossley, 2000) ou ainda, concebendo estágios de mudança, que combinam psicoterapia e estratégias de modificação de comportamento. Localizamos um artigo alinhado aos modelos ecológicos de promoção da saúde (Goh et al., 2009), modelos que, ao proporem mudanças comportamentais, destacam a interação entre o indivíduo e o seu ambiente social e físico (Parker et al., 2004).

Os trabalhos na perspectiva da primeira vertente adotavam diferentes construtos como "fatores psicossociais", como se pode observar no Quadro 1. Exceto nos dois últimos, a referência é o indivíduo.

Além dos construtos elencados na primeira coluna do Quadro 1, como "determinantes psicossociais", mencionam também preferências alimentares (Anderson-Bill et al., 2011; Tuuri et al., 2009; Van Duyn et al., 2001), resiliência (Clark et al., 2011), depressão (Clark et al., 2011; Zemper et al., 2003), problemas de ajustamento relacionados a perdas no trabalho e casamento e satisfação com a vida (Zemper et al., 2003). Um dos artigos mencionava aferição de fatores psicossociais em seu resumo, sem maior detalhamento (Rimmer et al., 2000).

Os trabalhos de intervenção analisados podem também ser categorizados de acordo com o seu alcance e modalidade: intervenções individuais que utilizam estratégias de promoção da saúde realizadas a distância, por computador, correio e/ou telefone (Anderson-Bill et al., 2011; Robroek et al., 2010; Elder et al., 2009; Portnoy et al., 2008; Van Keulen et al., 2008; Van Duyn et al., 2001), intervenções face a face realizadas com grupos específicos (Clark et al., 2011; Bonnel, 2003; Zemper et al., 2003; Langenberg et al., 2000; Rimmer et al., 2000), intervenções com base na família ou escola (Chen et al., 2010; Pearson et al., 2010; Goh et al., 2009; Tuuri et al., 2009; Burgess-Champoux et al., 2008; Haerens et al., 2008; Saksvig et al., 2005; Verheijden et al., 2003; Parcel et al., 2003) e, finalmente, intervenções de base comunitária (Gittelsohn et al., 2010; Mead et al., 2010; Campbell et al., 2007). 
Quadro 1. Construtos psicossociais nos trabalhos analisados

\begin{tabular}{|c|c|c|}
\hline Construto & Descrição & Modelo* \\
\hline $\begin{array}{l}\text { Attitude } \\
6 \text { trabalhos }\end{array}$ & $\begin{array}{l}\text { Atitude: é determinada pelas crenças individuais quanto a resultados ou atributos } \\
\text { da adoção de determinado comportamento }\end{array}$ & $\begin{array}{l}\text { TM } \\
\text { TPB } \\
\text { TRA }\end{array}$ \\
\hline $\begin{array}{l}\text { Awareness } \\
1 \text { trabalho }\end{array}$ & $\begin{array}{l}\text { Consciência: fator que influencia a motivação para a mudança de comportamento } \\
\text { e está relacionado à consciência sobre as causas de determinada doença ou agravo } \\
\text { em saúde, suas consequências e cura }\end{array}$ & TM \\
\hline $\begin{array}{l}\text { Knowledge } \\
8 \text { trabalhos }\end{array}$ & Conhecimento: sobre os riscos e benefícios de diferentes práticas & $\begin{array}{l}\text { SC } \\
\text { TM }\end{array}$ \\
\hline $\begin{array}{l}\text { Perceived health threat } \\
2 \text { trabalhos }\end{array}$ & $\begin{array}{l}\text { Risco percebido: combinação da percepção de suscetibilidade com a percepção de } \\
\text { severidade de determinada doença ou agravo em saúde }\end{array}$ & HBM \\
\hline $\begin{array}{l}\text { Positive outcome } \\
\text { expectations/negative } \\
\text { outcome expectations } \\
4 \text { trabalhos }\end{array}$ & $\begin{array}{l}\text { Expectativas de resultados positivos e negativos: estimativa pessoal de que o } \\
\text { comportamento irá gerar certos resultados }\end{array}$ & SC \\
\hline $\begin{array}{l}\text { Perceived benefits } \\
2 \text { trabalhos }\end{array}$ & $\begin{array}{l}\text { Benefícios percebidos: opinião quanto à eficácia da ação aconselhada na redução } \\
\text { de riscos para a saúde. }\end{array}$ & HBM \\
\hline $\begin{array}{l}\text { Perceived barriers } \\
4 \text { trabalhos }\end{array}$ & $\begin{array}{l}\text { Barreiras percebidas: opinião da pessoa sobre os custos materiais e não materiais } \\
\text { da ação aconselhada }\end{array}$ & $\begin{array}{l}\mathrm{HBM} \\
\mathrm{SC}\end{array}$ \\
\hline $\begin{array}{l}\text { Subjective norm } \\
1 \text { trabalho }\end{array}$ & $\begin{array}{l}\text { Normas subjetivas: expectativa em relação à aprovação (ou não) de outras pessoas } \\
\text { e desejo de aprovação (ou não). Também conhecido como suporte social } \\
\text { percebido. }\end{array}$ & TRA \\
\hline $\begin{array}{l}\text { Social norms } \\
3 \text { trabalhos }\end{array}$ & $\begin{array}{l}\text { Normas sociais: comportamentos valorizados e observados pelos indivíduos em seu } \\
\text { convívio social }\end{array}$ & $\begin{array}{l}\text { SC } \\
\text { TM }\end{array}$ \\
\hline $\begin{array}{l}\text { Self-efficacy/self } \\
\text { efficacy expectation } \\
17 \text { trabalhos }\end{array}$ & $\begin{array}{l}\text { Autoeficácia e expectativa de autoeficácia: confiança na capacidade de realizar a } \\
\text { mudança proposta. Afeta não só as metas que a pessoa propõe para si mesma como } \\
\text { outros determinantes, como os resultados que as pessoas esperam de sua ação. }\end{array}$ & $\begin{array}{l}\mathrm{HBM} \\
\mathrm{SC} \\
\mathrm{TM}\end{array}$ \\
\hline $\begin{array}{l}\text { Intention } \\
6 \text { trabalhos }\end{array}$ & Intenção: é determinada pela atitude e normas sociais & $\begin{array}{l}\text { TM } \\
\text { TPB } \\
\text { TRA }\end{array}$ \\
\hline $\begin{array}{l}\text { Self-regulation } \\
3 \text { trabalhos }\end{array}$ & $\begin{array}{l}\text { Autorregulação: capacidades autorreguladorasdas pessoas em afetar os seus } \\
\text { processos de pensamento, de motivação, de estados afetivos e de ações, por meio } \\
\text { da influência autodirigida. }\end{array}$ & $\begin{array}{l}\text { SC } \\
\text { TM }\end{array}$ \\
\hline $\begin{array}{l}\text { Skills } \\
\text { (habilidades) } \\
4 \text { trabalhos }\end{array}$ & $\begin{array}{l}\text { Habilidades: habilidades presentes ou que serão desenvolvidas para a mudança de } \\
\text { comportamento }\end{array}$ & SC \\
\hline $\begin{array}{l}\text { Family support } \\
1 \text { trabalho }\end{array}$ & $\begin{array}{l}\text { Suporte familiar: hábitos e comportamentos da família que ajudam a sustentar uma } \\
\text { alimentação adequada }\end{array}$ & SC \\
\hline $\begin{array}{l}\text { Social support } \\
8 \text { trabalhos }\end{array}$ & $\begin{array}{l}\text { Suporte social: encorajamento e apoio para o desenvolvimento de hábitos saudáveis } \\
\text { por pessoas da convivência do indivíduo }\end{array}$ & $\begin{array}{l}\text { SC } \\
\text { TM }\end{array}$ \\
\hline
\end{tabular}

TRA = Theory of Reasoned Action - Teoria da Ação Racional; TPB = Theory of Planned Behavior - Teoria do Comportamento Planejado; TM = Transtheoretical Model - Modelo Transteórico; HBM = Health Belief model - Modelo de Crenças em Saúde

"Modelos identificados a partir da abordagem explicitada nos artigos analisados e descrições dos construtos em Montaño, Kasprzyk e Taplin,

(2002), Prochaska, Redding e Evers (2002), e Strecher e Rosenstock (2002) 
Nesses trabalhos que visam promover mudanças no comportamento dos indivíduos, não questionam as condições sociais que tornam determinados indivíduos ou comunidades mais vulneráveis a piores condições de alimentação. Em intervenção descrita por Verheijden et al. (2003), por exemplo, que ofereceu aconselhamento nutricional familiar, os participantes avaliavam se alimentos com pouca gordura eram caros. Esse item do instrumento aferia a "atitude" dos indivíduos diante da proposta de mudança de comportamento. Como descrito no Quadro 1, a "atitude" dos indivíduos estaria relacionada às suas crenças. Nessa perspectiva a desigualdade de acesso aos alimentos com menos gordura que fundamenta essa crença deixa de ser abordada - apenas a crença é objeto da intervenção. Alternativamente, uma abordagem psicossocial construcionista, baseada em uma concepção de saúde implicada na proteção dos direitos humanos, essa questão diria menos sobre crenças do indivíduo e mais sobre o contexto objetivo que torna os indivíduos mais vulneráveis a piores condições de alimentação. Acredita-se que, ao não observar as condições concretas de produção da desigualdade, se corre o risco de responsabilizar o indivíduo que não adere às práticas propostas e perder de vista que aquele indivíduo vive em um contexto que não favorece que a saúde seja um recurso cotidiano.

O estudo-intervenção de Langenberg et al. (2000) com mulheres de baixa renda atendidas pelo Special Supplement Nutritional Program for Women (WIC) nos Estados Unidos, visava aumentar o consumo de frutas e vegetais, transformando os "fatores psicossociais" que impediriam a mudança do comportamento alimentar que seriam: a "atitude" das mulheres diante de frutas e vegetais, seu "nível de conhecimento" sobre as recomendações nacionais para o consumo desses alimentos, "barreiras percebidas" para o aumento do consumo e "sentimentos de autoeficácia" para fazer mudanças. A educação por pares (mulheres que já tinham participado do programa ou eram étnica ou socialmente do mesmo grupo das participantes) indica preocupação com a adequação e a especificidade do grupo abordado. Por outro lado, a condição de pobreza dessas mulheres não era tratada como um problema da ordem do social, mas como uma barreira que seria superável pela orientação. Se alimentos saudáveis eram caros, as educadoras deveriam fornecer estratégias para comer de maneira saudável mesmo em situação de pobreza. Assim, as "barreiras percebidas" não ajudavam a desvelar uma situação de vulnerabilidade social a piores condições de alimentação, mas eram abordadas com táticas para a mudança de comportamento individual, sem que sua existência fosse eticamente questionada. Para aquelas participantes, obter receitas e informações sobre frutas e vegetais mais acessíveis era importante e possível porém, quando os autores fazem apontamentos para as políticas públicas, sua proposta fundamenta-se na transmissão de informação, sem levar em conta a dificuldade de acesso a determinados alimentos. Focaliza-se a mudança das crenças, conhecimentos e, consequentemente, dos comportamentos, e as questões sociais não são abordadas.

Em estudo sobre efeitos de uma intervenção para um grupo de mulheres afro-americanas sobreviventes de derrame, Rimmer et al. (2000) observaram barreiras importantes aos objetivos de sua intervenção: custo elevado do programa, falta de transporte e de lugares em que as participantes pudessem se exercitar. No contexto do estudo essas barreiras foram eliminadas: o programa era gratuito, bem como o transporte para o local do estudo, onde as participantes fariam os exercícios com todos os recursos necessários, mas não se questionaram suas origens estruturais ou a necessidade de lidar diretamente com esse fato depois da intervenção. Ou seja, nessa perspectiva o fato de obstáculos socioestruturais impedirem a promoção da saúde no cotidiano das participantes não se transforma em desafio teórico-metodológico central, nem se discute o futuro do programa, sua institucionalização e sustentabilidade.

Indivíduos em situação de pobreza e vulnerabilidade social específica - sem trabalho, com baixo nível educacional, com filhos pequenos, pertencentes a grupos em que para superar essa condição enfrentam processos de discriminação adicional de direitos como as mulheres negras estudadas (ou ainda deficientes físicos ou homossexuais, como a literatura construcionista tem demonstrado) devem ser apoiados como pessoas concebidas como sujeitos de seu cotidiano e com direito a uma vida saudável para si e seus entes significativos. A compreensão da dimensão psicossocial com um olhar mais denso para a dimensão social permitiria aos profissionais envolvidos com a intervenção tratar dos limites estruturais, programáticos/institucionais que sustentam a maior probabilidade de alimentação inadequada desses segmentos. Essa vertente de intervenções não inclui a noção de que essas pessoas 
${ }^{5} \mathrm{O}$ "direito à alimentação adequada" implica garantir aos indivíduos e grupos que estejam impossibilitados de usufruir o direito à alimentação adequada a provisão desse direito diretamente pelos Estados. devem ser acolhidas e cuidadas como sujeitos do direito à alimentação adequada ${ }^{5}$ - direito não garantido nos Estados Unidos, país em que a maior parte desses modelos foi testada, apesar de sua equiparação aos demais direitos do homem e reconhecimento de que todos os Estados têm de respeitar, proteger e realizar esse direito (Belik, 2003).

A questão da desigualdade social e seus efeitos objetivos não é abordada nem na discussão dos limites das intervenções. Anderson-Bill et al. (2011), por exemplo, realizaram uma intervenção a distância, pela internet, e excluíram de sua amostra pessoas com contraindicação para a realização de atividades físicas (idade avançada, problemas de saúde e obesidade mórbida). Os autores destacam que a maior parte dos interessados excluídos do estudo eram negros, aspecto não analisado no artigo apesar de a literatura norte-americana, mais até do que a brasileira, discutir com frequência como raça e etnia estão implicadas no processo saúde-doença, reconhecendo uma história social, e não apenas uma história individual ou natural, atribuível à cor da pele (Ayres, Paiva, França Júnior, 2011).

Já no caso das intervenções realizadas em escolas (Tuuri et al., 2009; Haerens et al., 2008; Saksvig et al., 2005; Parcel et al., 2003), partiu-se da necessidade de abordar a instituição e, portanto, membros da comunidade escolar foram incluídos na proposição e avaliação das intervenções, mostrando clara preocupação com a dimensão programática e com a participação da comunidade. Nessa direção, chama a atenção o estudo de Haerens et al. (2008), realizado na Bélgica, que considera relevante a sustentabilidade do programa, preparando os funcionários da escola para realizá-lo, algo fundamental para a efetiva transformação programática. Porém, o limite da intervenção é ainda apenas o espaço escolar e as avaliações são feitas com base em dados individuais (com aferição de "determinantes psicossociais") e dentro do espaço escolar ("clima" da escola), não se considerando o necessário imbricamento dinâmico de questões socioeconômicas e políticas (estruturais e programáticas) que ampliaria a compreensão dinâmica da comunidade para além do espaço próximo.

Trabalhos realizados com famílias (Chen et al., 2010; Pearson et al., 2010; Burgess-Champoux et al., 2008; Verheijden et al., 2003) expressam essa mesma perspectiva. Justificam-se intervenções no ambiente familiar para que crianças possam ter um ambiente no qual a alimentação saudável é estimulada: frutas e vegetais ficariam à disposição e pais atuariam como modelos para que a criança aprenda aqueles comportamentos. Porém, será que os pais dessas crianças têm, eles mesmos, acesso a frutas e vegetais? Teriam consciência da ação cotidiana dos fortes interesses políticos e econômicos que, pela televisão, produzem a cultura do "valor" de seus produtos industrializados? O contexto social mais amplo não é abordado e, mais importante, não se introduz como parte das informações a serem discutidas a noção de que o meio social é resultado da produção de homens e mulheres ao longo da história. Na concepção da vertente psicossocial construcionista, por outro lado, o "meio" não pode ser tratado como um meio natural, mas algo a ser também modificado por indivíduos concebidos como sujeitos portadores de direitos.

Os trabalhos de base comunitária, de qualquer modo, demonstram maior cuidado na adaptação da intervenção à cultura local dos participantes e ressaltam a importância de envolver efetivamente a comunidade da qual esses participantes fazem parte. Não são, entretanto, muito diferentes quando definem determinantes "psicossociais" como características da dimensão individual e naturalizam (porque não questionam) as suas determinações macrossociais.

Alguns pesquisadores usam a noção de "empowerment" (Clark et al., 2011; Goh et al., 2009) que, especialmente nos EUA, significa empoderamento 
individual, fortalecer o poder da vontade, com pouca referência à história de produção da desigualdade social (Paiva, 1996). Um agente externo, um profissional de saúde, empodera indivíduos e comunidades oferecendo informações e treinamento para que possam promover mudanças em suas vidas e pretende que a intervenção transforme indivíduos em senhores de sua vida cotidiana, adotando práticas alimentares, ou atividades físicas pré-definidas como mais saudáveis, desconsiderando as desigualdades sociais, de gênero e raciais que atravessam as práticas e cotidianos dos participantes - e dos profissionais de saúde - nas ações de promoção de práticas alimentares saudáveis. Avalia-se que indivíduos que não adequam seus comportamentos durante a intervenção não estariam preparados para a mudança (estariam em estágio inicial) ou que não aprenderam como deveriam, ou ainda, que não há nada a fazer - a não ser criar condições ideais, controlar fatores que atrapalham a eficácia da intervenção sem maior preocupação com sua sustentabilidade ou institucionalização.

Poucos artigos enfatizam o contexto mais amplo da intervenção que realizaram ou a impossibilidade de generalização de seus achados (Gittelsohn et al., 2010; Pearson et al., 2010; Elder et al., 2009) e consideram de maneira mais interessante a dimensão social na análise, indicando certa elaboração quanto a obstáculos que excedem o plano individual. Elder et al. (2009) incluem na discussão sobre intervenção com mulheres latinas vivendo nos Estados Unidos a consideração de que barreiras econômicas, sociais e outras "barreiras ambientais" poderiam ter desencorajado as mulheres a manter as mudanças comportamentais introduzidas pela intervenção. Reconhecem barreiras que são limites do contexto e não de "capacidades" ainda não desenvolvidas pelo indivíduo. Pearson et al. (2010), em trabalho de promoção da saúde com pais e jovens, têm o cuidado de mencionar que sua intervenção, baseada no envio de material impresso, atingiu pessoas com bom nível educacional, e que seus resultados não podem ser generalizados.

Finalmente, entre os trabalhos mais atentos à dimensão estrutural e programática, destacamos a intervenção de Gittelsohn et al. (2010), que procurou provocar alterações nos fatores psicossociais dedicando-se ao contexto: os autores promoveram mudanças na oferta, preços e divulgação de alimentos mais saudáveis em lojas e supermercados de uma comunidade de baixa renda, acompanhadas de intervenções pontuais com o público que frequentava essas lojas. Não conseguiram medir alteração significativa nos fatores psicossociais avaliados pelo estudo ("conhecimento", "autoeficácia" e "intenção"). Destacamos que, apesar da relevância de intervenções como essa, a comunidade não participou da construção da intervenção e, aparentemente, a relação entre os indivíduos e contexto social, mediada por crenças (na perspectiva da abordagem sociocognitiva), é naturalizada: não são considerados sentidos e significados intersubjetivamente construídos, alteram-se preços e divulgação de alimentos mais saudáveis e espera-se que os indivíduos passem a conhecer mais, se sintam mais capazes e fortaleçam a intenção de consumir esses alimentos.

\section{Discussão}

Como na crítica ao modelo de HND na década de 1970, as ações de promoção de práticas alimentares saudáveis analisadas no presente trabalho apresentaram, em geral, uma concepção da dimensão psicossocial do processo saúde-doença que naturaliza processos sociais e políticos que coproduzem a vulnerabilidade a piores condições de alimentação. Os determinantes sociais e psicológicos do processo saúde-doença são considerados, mas o indivíduo ainda é concebido e abordado como unidade biológica-comportamental, sem que se interpele a construção social dos determinantes do processo saúde-doença (Paiva, 2009, 2006). O contexto social é importante enquanto informa predisposições, hábitos, pensamentos e intenções individuais com influência mensurável sobre os comportamentos indicados como adequados, mas não é visto como uma construção históricopolítica. Assim, quem deve mudar é o indivíduo. Ou o ambiente, tomado como espaço esvaziado de intenções e disputas políticas, deve ser alterado para favorecer comportamentos específicos.

O foco na mudança individual de comportamentos, pressupondo que a adoção de práticas alimentares mais saudáveis depende apenas de ações individuais, não é exclusiva dos modelos que compreendem a dimensão psicossocial dessa forma. Essa concepção é frequente e está disseminada 
entre profissionais de saúde e entre as pessoas que gostariam de se alimentar melhor. Lara (2010), em trabalho com um grupo de ajuda mútua para a discussão de peso, alimentação e saúde, relata que as mulheres que participavam do grupo acreditavam que emagrecer só dependia delas e de sua força de vontade, bem como alguns profissionais de saúde que atendiam essas mulheres demonstravam esse tipo de posicionamento. Não há intenção de enfrentar dificuldades impostas pelo contexto, como, por exemplo, a má qualidade da merenda servida na escola. Pelo contrário, diante de inadequações do contexto, é o indivíduo que deve ser "criativo" e se adaptar. Nos trabalhos analisados, mesmo quando se levavam em conta construtos como "barreiras percebidas", o enfoque era adaptativo: o indivíduo é ensinado a conviver com as barreiras e contorná-las da melhor maneira possível, sem que sua existência seja questionada, como se as barreiras fossem naturais e inevitáveis, e não resultado de ação humana.

Seguindo essa linha, os trabalhos que abordam a dimensão psicossocial nessa vertente dos estilos de vida enfatizam que as pessoas podem mudar suas práticas alimentares se tiverem o conhecimento e habilidade necessárias, o que sabemos não ser suficiente quando se trata de alimentação. Pessoas que aprenderam como deveriam se alimentar e estratégias para manter uma boa alimentação podem enfrentar dificuldades das mais diversas ordens, mas seu conhecimento e habilidades não garantirão a manutenção de uma boa alimentação. Esse tipo de compreensão de processo psicossocial pode levar à culpabilização e, eventualmente, à desesperança especialmente aqueles que não têm condições sociais de realizar as mudanças precisas desenhadas pelos programas. Lara (2010) discute que algumas pessoas sentiam vergonha por não estarem conseguindo mudar suas práticas alimentares e isso fazia com que faltassem às reuniões do grupo ou desistissem de participar.

Assim, embora esses modelos de abordagem da dimensão psicossocial reconheçam que disposições, cognições e crenças dos indivíduos são influenciadas por determinantes sociais e psicológicos, não avançam na compreensão de que as pessoas participam ativamente da construção dos contextos. Sem essa compreensão, os contextos continuam sendo compreendidos à maneira ecológica, como ambientes que podem ser alterados para promover saúde mas que, em última instância, não guardam qualquer vestígio das relações sociais, políticas e econômicas sobre as quais historicamente se estruturaram. Não é por outro motivo que as estratégias são fundamentalmente psicoeducativas, voltadas a ampliar o conhecimento a respeito dos riscos e benefícios de seus comportamentos e desenvolver habilidades específicas, melhorar a atitude, apoiar intenções e promover melhora da autoeficácia, como observamos nas principais estratégias de promoção da saúde do modelo de HND.

Um limite do presente trabalho é o fato de que o caminho que a busca pela literatura seguiu privilegiou a literatura norte-americana (especialmente estudos realizados nos Estados Unidos) ou de países desenvolvidos, como Reino Unido, Bélgica e Holanda, onde encontramos estudos que declaradamente faziam referência a aspectos ou a uma dimensão psicossocial. Outros estudos são necessários para avaliar se há outros enfoques para a dimensão psicossocial em intervenções promotoras de práticas alimentares saudáveis que não necessariamente utilizem o termo "psicossocial". Talvez outras nomenclaturas estejam em uso em diferentes correntes teóricas, de maneira que essa avaliação se faz necessária. Para o presente trabalho, interessava conhecer a concepção de dimensão psicossocial que estava fundamentando intervenções promotoras de práticas alimentares saudáveis, mas há trabalhos que não utilizam o termo "psicossocial" e que certamente pensam o indivíduo em sociedade, sendo necessário realizar uma revisão mais ampla de estratégias de promoção da saúde no campo da alimentação para avaliar como outras abordagens têm abordado a interação entre indivíduo e sociedade.

\section{Uma outra possibilidade: fortalecer a vertente construcionista}

Para trabalhar com a promoção de práticas alimentares saudáveis no paradigma da MPS, a vertente psicossocial construcionista parece mais interessante para a intervenção no processo saúde-doençacuidado. A vertente psicossocial construcionista reconheceu a virada epistemológica validada desde o século XX, que concebe a vida social como produzida historicamente pelos homens e mulheres que nele habitam; e reconhece que o "meio" é atravessado por desigualdades sociais de diversas ordens até então naturalizadas (de gênero, de raça, de idade); reconhece que relações desiguais são sustentadas 
por uma rede de significados, sentidos e práticas discursivas que, assim como foram construídos pela ação humana, podem ser reconstruídos e resignificados. Mais importante, tem demonstrado em outros campos que essa é uma referência conceitual e ético-política relevante para informar as técnicas e práticas, e a ser disseminada também para participantes de programas de promoção da saúde.

Caminha para uma mais forte politização de suas ações, em contraposição ao modelo de HND, no qual predomina, como vimos, uma leitura mais técnica e individual dos problemas e de suas soluções (Ayres, 2002).

Mais especificamente, entre as possibilidades dessa vertente, destacamos a compreensão da dimensão psicossocial no quadro da vulnerabilidade e dos direitos humanos, concebida como sinergia de três dimensões mutuamente implicadas na produção do adoecer e na promoção da saúde: a dimensão individual, a social e a programática da vulnerabilidade ao sobrepeso, por exemplo (Paiva, Ayres, Gruskin, 2010; Paiva, 2009, 2006; Ayres et al., 2006; Ayres et al., 2003; Berkman et al., 2005), como ilustrado na Figura 1.

Figura 1. Dimensões individual, social e programática de vulnerabilidade à insegurança alimentar - dimensões necessariamente implicadas

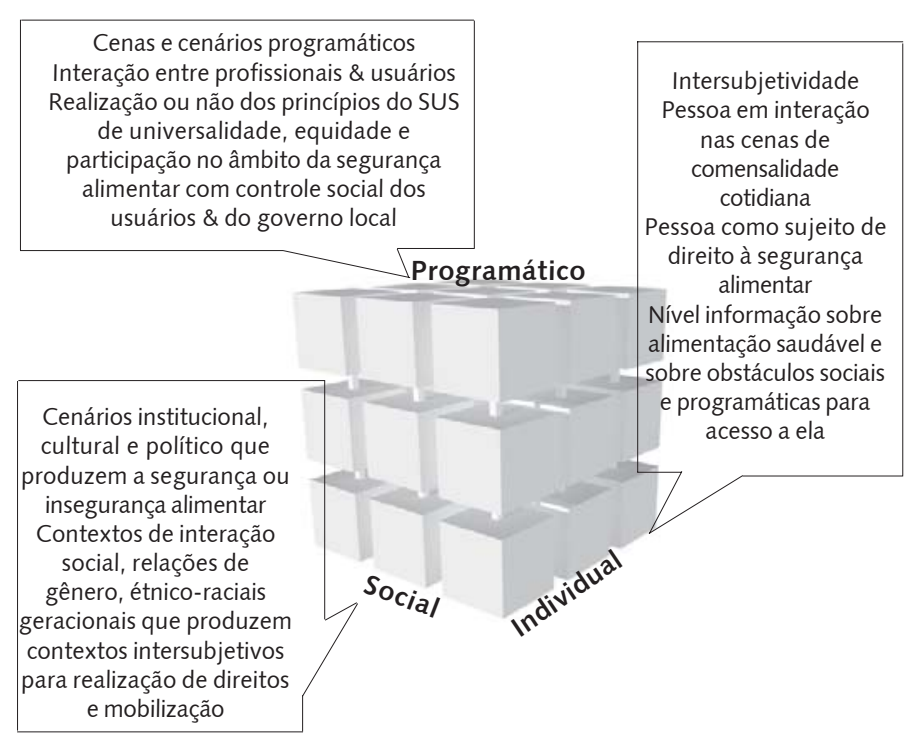

Adaptado de Paiva, Ayres e Gruskin (2010).

O plano individual é definido como o plano intersubjetivo e da pessoa que é sujeito de direitos, não existindo separado do plano social ou programático, assim como a pessoa não existe fora de seu contexto (Paiva, 2009). A abordagem psicossocial nesse quadro desafia-nos a reconhecer que, para a pessoa em condições de grande vulnerabilidade social e individual, a presença e ação de um programa de qualidade são fundamentais, como pudemos observar em programas brasileiros nos campos da aids e da saúde da mulher.

A desigualdade é considerada elemento central na produção do processo saúde-doença, estabelecendo-se estreito diálogo com o quadro dos direitos humanos (França Júnior, Ayres, 2003), observável nas existências intersubjetivas dos usuários com programas de saúde ou em sua vida cotidiana. Como discutiram Kalichman e Diniz (2009), seu sucesso depende da validação do direito à saúde como direito universal, como se fez no caso da aids, algo mais difícil de ser compreendido em países nos quais a reforma sanitária não consegue se estabelecer. 
A concepção de indivíduo dessa perspectiva significa a pessoa como sujeito do direito à saúde integral e à alimentação adequada. O desenho de uma intervenção psicossocial no campo das práticas alimentares nesse quadro valorizará o saber prático dos sujeitos da intervenção, porque usuários são os maiores especialistas em sua vida cotidiana e contexto específico, contexto que pode mudar para além do espaço próximo.

No Quadro 2 estabelecemos uma comparação entre os princípios da MPS e a proposta de abordagem da dimensão psicossocial no quadro da vulnerabilidade e dos direitos humanos. Essa perspectiva alinha-se mais adequadamente à MPS do que os modelos classificados na vertente psicossocial focada em estilos de vida.

Em condições concretas de desigualdade na realização de direitos fundamentais como o direito à alimentação adequada, saúde integral, ao cuidado e à prevenção, é responsabilidade de programas de saúde e seus profissionais (e não do indivíduo apenas) garantir menor vulnerabilidade social e individual à obesidade ou à desnutrição. Especialmente em contextos de desigualdade extrema, abordagens focadas em estilo de vida e mudança de comportamento dos indivíduos terão pouca efetividade.

Quadro 2. Princípios da moderna Promoção da Saúde e estratégias compatíveis de redução da vulnerabilidade a partir de uma abordagem psicossocial construcionista baseada no quadro da vulnerabilidade e dos direitos humanos

\begin{tabular}{|c|c|}
\hline Promoção da Saúde & Redução de Vulnerabilidade \\
\hline $\begin{array}{l}\text { Concepção holística de saúde voltada para a multicausalidade: } \\
\text { são incorporados, para a compreensão do processo saúde- } \\
\text { doença, os determinantes sociais, econômicos, ambientais, e } \\
\text { não só os biológicos, que não agem isoladamente, mas } \\
\text { interagem uns com os outros. }\end{array}$ & $\begin{array}{l}\text { O processo saúde-doença tem múltipla causalidade e } \\
\text { três dimensões a serem enfrentadas: individual, socia } \\
\text { e programática, que interagem na produção de } \\
\text { contextos de maior vulnerabilidade ao adoecimento } \\
\text { e/ou ao agravo de saúde. }\end{array}$ \\
\hline $\begin{array}{l}\text { Equidade: garantir o acesso universal à saúde e eliminar as } \\
\text { diferenças desnecessárias e injustas que restringem as } \\
\text { oportunidades para se atingir o bem-estar. }\end{array}$ & $\begin{array}{l}\text { Proteção e promoção dos direitos humanos/ Pessoas } \\
\text { concebidas como sujeitos de direito }\end{array}$ \\
\hline $\begin{array}{l}\text { Intersetorialidade: ao lidar com a multicausalidade dos } \\
\text { problemas de saúde reconhece a limitação do setor saúde e } \\
\text { coloca a necessidade de múltiplos olhares e saberes. }\end{array}$ & $\begin{array}{l}\text { Intersetorialidade das ações, que dependem de } \\
\text { diferentes saberes e do envolvimento de outros } \\
\text { setores além do setor saúde. }\end{array}$ \\
\hline $\begin{array}{l}\text { Ações multiestratégicas: pressupõe o envolvimento de } \\
\text { diferentes disciplinas e combinação de métodos e abordagens. }\end{array}$ & $\begin{array}{l}\text { Ações que dependem da intersetorialidade e da } \\
\text { multidisciplinaridade }\end{array}$ \\
\hline $\begin{array}{l}\text { Participação social: pessoas como colaboradoras diretas e com } \\
\text { direito a participar das decisões envolvendo sua saúde. }\end{array}$ & $\begin{array}{l}\text { Atitude construcionista/ processo colaborativo desde } \\
\text { a identificação do problema e necessidades até a } \\
\text { avaliação conjunta dos resultados das ações }\end{array}$ \\
\hline $\begin{array}{l}\text { Empoderamento: processo de capacitação dos indivíduos e } \\
\text { comunidades para assumirem maior controle sobre os fatores } \\
\text { pessoais, socioeconômicos e ambientais que afetam a saúde. }\end{array}$ & $\begin{array}{l}\text { Autonomia/Favorecer a capacidade de resposta/ } \\
\text { Emancipação de uma situação de vulnerabilidade }\end{array}$ \\
\hline $\begin{array}{l}\text { Sustentabilidade; iniciativas que estejam de acordo com os } \\
\text { princípios do desenvolvimento sustentável e que garantam um } \\
\text { processo duradouro e forte. }\end{array}$ & $\begin{array}{l}\text { Sustentabilidade das ações pensada a partir do } \\
\text { compromisso e responsabilização de governos pela } \\
\text { proteção e promoção de direitos }\end{array}$ \\
\hline
\end{tabular}

Referências: Ayres (2009); Paiva (2009, 2006, 2002, 1999); Ayres et al. (2006, 2003); Westphal (2006); Sícoli e Nascimento (2003).

\section{Considerações finais}

Na literatura analisada (2000-2011), que cobriu intervenções promotoras de práticas alimentares saudáveis atentas à dimensão psicossocial, buscou-se descrever como concebiam essa dimensão muito citada e pouco definida. Os modelos cognitivos e sociocognitivos foram mais frequentes, e sua compreensão da dimensão psicossocial do processo saúde-doença os classificaria na vertente 
psicossocial que enfatiza os estilos de vida na cadeia multicausal dos processos de adoecimento. Suas ações visam comportamentos individuais e o contexto social é concebido como "ambiente" no sentido de uma ecologia e geografia sem pessoas, cujos sentidos e significados são naturalizados.

Tal como são manejados, os construtos utilizados nesses modelos têm uma concepção mais estreita do que necessário da dimensão psicossocial do processo saúde-doença-cuidado. A dimensão social é levada em conta como formadora de comportamentos e cognição, predisposições e obstáculos que serão trabalhados apenas no âmbito individual. Na maior parte dos trabalhos analisados, sua construção social, política e histórica não é abordada.

Embora em alguns trabalhos haja referência à necessidade de ação por meio da organização social, a leitura dos problemas e soluções resulta em passos a serem seguidos para obtenção de êxito técnico no plano individual, contemplando especialmente atividades educativas e comportamentais, aproximando as intervenções propostas às práticas de saúde no modelo de HND.

Finalmente, para aprofundar o diálogo com a MPS, a vertente que aborda a dimensão psicossocial desde uma perspectiva construcionista e que pensa o processo saúde-doença baseada no quadro da vulnerabilidade e dos direitos humanos parece mais interessante para pensar na promoção de mudanças sustentáveis em contextos objetivos e intersubjetivos marcados pela desigualdade e desrespeito aos direitos humanos, em especial o direito à saúde integral.

\section{Colaboradores}

As autoras trabalharam juntas em todas as etapas de produção do manuscrito.

\section{Referências}

ANDERSON-BILL, E.S. et al. Web-based guide to health: relationship of theoretical variables to change in physical activity, nutrition and weight at 16-months. J. Med. Internet Res., v.13, n.1, p.e27, 2011.

AYRES, J.R.C.M.; PAIVA, V.S.F.; FRANÇA JR., I. From natural history of disease to vulnerability: changing concepts and practices in contemporary public health. In: PARKER, R.; SOMMER, M. Routledge handbook in global public health. Oxon: Taylor and Francis, 2011. p.98-107.

AYRES, J.R.C.M. Prevenção de agravos, promoção da saúde e redução de vulnerabilidade. In: MARTINS, M. et al. Atuação da Clínica Médica, sinais e sintomas de natureza sistêmica, medicina preventiva, saúde da mulher, envelhecimento e geriatria, medicina laboratorial e prática médica. Barueri: Manole, 2009. p.437-55. (Clinica Médica, v.1).

Epidemiologia, promoção da saúde e o paradoxo do risco. Rev. Bras. Epidemiol., v.5, supl.1, p.28-42, 2002.

AYRES, J.R.C.M. et al. Vulnerability, Human Rights, and Comprehensive Health Care Needs of Young People Living With HIV/AIDS. Am. J. Public Health, v.96, n.6, p.1001-6, 2006.

O conceito de vulnerabilidade. In: CZERESNIA, D.; FREITAS, C.M. Promoção da Saúde: conceitos, reflexões, tendências. Rio de Janeiro: Fiocruz, 2003. p.117-39.

BELIK, W. Perspectivas para segurança alimentar e nutricional no Brasil. Saude Soc. v.12, n.1, p.12-20, 2003.

BENZAKEN, A.S. et al. Intervenção de base comunitária para a prevenção das DST/Aids na região amazônica, Brasil. Rev. Saude Publica, v.41, n.2, p.118-26, 2007. 
BERKMAN, A. et al. A critical analysis of the Brazilian rto HIV/Aids: lessons for controlling and mthe epidemic in developing countries. Am. J. Public Health, v.95, n.7, p.1162-72, 2005.

BONNEL, W. Nutritional health promotion for older adults, where is the content? J. Am. Acad. Nurse Pract., v.15, n.5, p.224-9, 2003.

BRASIL. Informe: revisão da Política Nacional de Alimentação e Nutrição no SUS: PNAN 10 anos. 2010. Disponivel em: <http://portal.saude.gov.br/portal/arquivos/ pdf/pnan_020810.pdf>. Acesso em: 10 nov. 2012.

BURGESS-CHAMPOUX, T.L. et al. The development of psychosocial measures for whole-grain intake among children and their parents. J. Am. Diet. Assoc., v.108, n.4, p.714-7, 2008.

CAMPBELL, M.K. et al. Process evaluation of an effective church-based diet intervention: body \& soul. Health Educ. Behav., v.34, n.6, p.864-80, 2007.

CHEN, J.L. et al. The active balance childhood program for improving coping and quality of life in Chinese American children. Nurs. Res., v.59, n.4, p.270-9, 2010.

CLARK, P.G. et al. Maintaining exercise and healthful eating in older adults: the senior project II: study design and methodology. Contemp. Clin. Trials, v.32, n.1, p.129-39, 2011.

CROSSLEY, M.L. Rethinking psychological approaches to health promotion. In: (Org.). Rethinking health psychology. Buckingham: Open University Press, 2000. p.36-62.

ELDER, J.P. et al. Evaluating psychossocial and behavioral mechanisms or change in a tailored communication intervention. Health Educ. Behav., v.36, n.2, p.366-80, 2009

FRANÇA JR., I.; AYRES, J.R.C.M. Saúde pública e direitos humanos. In: ZÓBOLI, E.; FORTES, P. (Org.). Bioética e saúde pública. São Paulo: Loyola, 2003. p.63-9.

GITTELSOHN, J. et al. An urban food store intervention positively affects food-related psychosocial variables and food behaviors. Health Educ. Behav., v.37, n.3, p.390-402, 2010.

$\mathrm{GOH}$, Y.Y. et al. Using community-based participatory research to identify potential interventions to overcome barriers to adolescent's healthy eating and pshyscal activity. J. Behav. Med., v.32, n.5, p.491-502, 2009.

GRUSKIN, S.; TARANTOLA, D. Universal access to HIV prevention, treatment, and care: assessing the inclusion of human rights in international and national strategic plans. AIDS, v.22, n.2, p.S123-2, 2008.

HAERENS, L. et al. Explaining the effect of a 1-year intervention promoting physical activity in middle schools: a mediation analysis. Public Health Nutr., v.11, n.5, p.501-2, 2008

KALICHMAN, A.O.; DINIZ, S.G. Aids treatment in Brazil: what kind of evidence do we need?. Lancet, v.374, n.9695, p.1066, 2009.

LANGENBERG, P. et al. Psychosocial factors and intervention-associated changes in those factors as correlates of change in fruit and vegetable consumption in the Maryland WIC 5 a day promotion program. Ann. Behav. Med., v.22, n.4, p.307-15, 2000.

LARA, B.R. Uma abordagem psicossocial na promoção da saúde: refletindo criticamente sobre o processo de um grupo de apoio e discussão sobre peso e alimentação. 2010. Dissertação (Mestrado) - Instituto de Psicologia, Universidade de São Paulo, São Paulo. 2010.

MANN, J.; TARANTOLA, D.M.J. Aids in the world II. 2.ed. Oxford: Oxford University Press, 1996. 
MEAD, E. et al. Important psychosocial factors to target in nutrition interventions to improve diet in Inuvialuit communities in the Canadian Arctic. J. Hum. Nutr. Diet., v.23, n.1, p.92-9, 2010.

MONTAÑO, D.E.; KASPRZYK, D.; TAPLIN, S.H. The theory of reasoned action and the theory of planned behavior. In: GLANZ, K.; RIMMER, B.K.; VISWANATH, K. (Orgs.). Health behavior and health education: theory, research and practice. 2.ed. San Francisco: Jossey-Bass, 2002. p.85-112.

PAIVA, V.S.F. A dimensão psicossocial do cuidado. In: NEPAIDS. Apostila do Curso de Especialização em Prevenção ao HIV/Aids no Quadro da Vulnerabilidade e dos Direitos Humanos. São Paulo: Nepaids, 2010. [mimeogr.]

Prevenção PositHIVa: a abordagem psicossocial, emancipação e vulnerabilidade. In: RAXACH, J.C. et al. (Orgs.). Prevenção Posithiva: estado da arte. Rio de Janeiro: ABIA, 2009. p.33-49.

Analisando cenas e sexualidades: a promoção da saúde na perspectiva dos direitos humanos. In: CÁCERES, C.F. et al. (Orgs.). Sexualidad, estigma y derechos humanos: desafios para el acceso a la salud en America Latina. Lima: Universidad Peruana Cayetano Heredia, 2006. p.23-51.

Analysing sexual experiences through "scenes": a framework for the evaluation of sexuality education. Sex. Educ., v.5, n.4, p.345-58, 2005.

Sem mágicas soluções: a prevenção e o cuidado em HIV/Aids e o processo de emancipação psicossocial. Interface - Comunic., Saude, Educ., v.6, n.11, p.25-38, 2002.

Cenas sexuais, roteiros de gênero e sujeito sexual. In: BARBOSA, R.M.; PARKER, R. (Orgs.). Sexualidades pelo avesso: direitos, identidades e poder. Rio de Janeiro: IMS/UERJ, 1999. p.250-71.

Sexualidades adolescentes: escolaridade, gênero e o sujeito sexual. In: PARKER, R.; BARBOSA, R.M. (Orgs.). Sexualidades brasileiras. Rio de Janeiro: Relume Dumará, 1996. p.213-34.

PAIVA, V.S.F.; AYRES, J.R.C.M.; FRANÇA JÚNIOR, I. Expanding the flexibility of normative patterns in youth sexuality and prevention and care programs. Sex. Res. Soc. Policy, v.1, n.1, p.1-15, 2004.

PAIVA, V.S.F.; AYRES, J.R.C.M.; GRUSKIN, S. Being young and living with HIV: the double neglect of sexual citizenship. In: AGGLETON, P.; PARKER, R. (Eds.). The routledge handbook of sexuality, health and rights. Londres: Routledge, 2010. p.422-32.

PAIVA, V.S.F. et al. Religious communities and HIV prevention: an intervention study using a human rights-based approach. Glob. Public Health, v.5, n.3, p.280-94, 2010.

PARCEL, G.S. et al. School climate and the institutionalization of the CATCH program. Health Educ. Behav., v.30, n.4, p.489-502, 2003.

PARKER, E.A. et al. Application of health promotion theories and models for environmental health. Health Educ. Behav., v.31, n.4, p.491-509, 2004

PEARSON, N. et al. A family-based intervention to increase fruit and vegetable consumption in adolescents: a pilot study. Public Health Nutr., v.13, n.6, p.876-85, 2010

PORTNOY, D.B. et al. Computer-delivered interventions for health promotion and behavioral risk reduction: a meta-analysis of 75 randomized controlled trials, 1988-2007. Prev. Med., v.47, n.1, p.3-16, 2008. 
PROCHASKA, J.O.; REDDING, C.A.; EVERS, K.E. The transtheoretical model and stages of change. In: GLANZ, K.; RIMMER, B.K.; VISWANATH, K. (Orgs.). Health behavior and health education: theory, research and practice. 2.ed. San Francisco: Jossey-Bass, 2002. p.60-84

RIMMER, J.H. et al. Effects of a short-term health promotion intervention for a predominantly African-American group of stroke survivors. Am. J. Prev. Med., v.18, n.4, p.332-8, 2000.

ROBROEK, S.J.W. et al. Demographic, behavioral, and psychosocial correlates of using the website component of a worksite physical activity and healthy nutrition promotion program: a longitudinal study. J. Med. Internet Res., v.12, n.3, p.e44, 2010.

SAKSVIG, B.I. et al. A pilot school-based healthy eating and physical activity intervention improves diet, food knowledge, and self-efficacy for native Canadian children. J. Nutr., v.135, n.10, p.2392-8, 2005.

SANTOS, M.A. Análise crítica de uma intervenção para melhoria da adesão do paciente vivendo com aids à terapia antirretroviral. 2011. Dissertação (Mestrado) Instituto de Psicologia, Universidade de São Paulo, São Paulo. 2011.

SíCOLI, J.L.; NASCIMENTO, P. R. Promoção da saúde: concepção, princípios, operacionalização. Interface - Comunic., Saude, Educ., v.7, n.12, p.91-112, 2003.

SPINK, M.J.P.A construção social do saber sobre saúde doença: uma perspectiva psicossocial. Saude Soc., v.1, n.2, p.125-39, 1992.

STRECHER, V.J.; ROSENSTOCK, I.M. The health belief model. In: GLANZ, K.; RIMMER, B.K.; VISWANATH, K. (Orgs.). Health behavior and health education: theory, research and practice. 2.ed. San Francisco: Jossey-Bass, 2002. p.41-59.

TRAVERSO-YÉPES, M.A. Dilemas na promoção da saúde no Brasil: reflexões em torno da política nacional. Interface - Comunic., Saude, Educ., v.11, n.22, p.223-38, 2007.

TUNALA-MENDONÇA, L. G. Promoção da saúde: análise do processo de um grupo de reeducação alimentar em um hospital universitário. 2005. Tese (Doutorado) - Instituto de Psicologia, Universidade de São Paulo, São Paulo. 2005.

TUURI, G. et al. 'Smart bodies' school wellness program increased children's knowledge of healthy nutrition practices and self-efficacy to consume fruit and vegetables.

Appetite, v.52, n.2, p.445-51, 2009.

VAN DUYN, M.A. et al. Association of awareness, intrapersonal and interpersonal factors, and stage of dietary change with fruit and vegetable consumption: a national survey. Am. J. Health Promot., v.16, n.2, p.69-78, 2001.

VAN KEULEN, H. M. et al. Vitalum study design: RCT evaluating the efficacy of tailored print communication and telephone motivational interviewing on multiple health behaviors. BMC Public Health, v.19, n.8, p.216, 2008

VERHEIJDEN, M.W. et al. Nutrition guidance in Dutch family practice: behavioral determinants of reduction of fat consumption. Am. J. Clin. Nutr., v.77, supl.4, p.1058S-64S, 2003.

WESTPHAL, M.F. Promoção da saúde e prevenção de doenças. In: CAMPOS, G.W.D.S. (Org.). Tratado de saúde coletiva. São Paulo: Hucitec, 2006. p.635-67.

ZEMPER, E.D. et al. Assessment of a holistic wellness program for persons with spinal cord injury. Am. J. Phys. Med. Rehab., v.82, n.12, p.957-68, 2003. 
LARA, B.R.; PAIVA, V.S.F. La dimensión psico-social en la de promoción de prácticas alimentarias saludables. Interface - Comunic., Saude, Educ., v.16, n.43, p.1039-54, out./dez. 2012.

Este artículo analiza críticamente como la dimensión psico-social es abordada en la promoción de prácticas alimentarias saludables. Se realizó una búsqueda en LILACS y en el modo multi-propósito de la Medline, de 2000 a 2011, utilizando los términos intervención, promoción de la salud, psico-social, y todos los términos correlatos a nutrición. Se observó que en esta última década los enfoques socio-cognitivos y modelos de creencia racional predominan en este campo, prevaleciendo trabajos de intervención focalizados en el individuo y poco críticos en relación al contexto social que produce prácticas alimentarias. Se concluyó que para la promoción de prácticas alimentarias saludables en el contexto de la atención integral, el debate sobre lo psicosocial debe ser ampliado para incorporar las contribuciones recientes de los enfoques en salud basados en los derechos humanos, atentos a la multi-dimensión del proceso salud-cuidado.

Palabras clave: Promoción de la salud. Psico-social. Cuidado. Educación alimentaria y nutricional. Derechos humanos. 